D0I: 10.12957/demetra.2016.18445

\title{
Elaboração e avaliação da aceitabilidade do fishburger de acará-açu (Lobotessurinamensis) no mercado macapaense - AP, Brasil
}

\section{The elaboration and the evaluation of the acceptability of the fish burger of acará-açú (Lobotessurinamensis) in the macapaense market - AP, Brazil}

\author{
Marilu Teixeira Amaral ${ }^{1}$ \\ Fredson Costa Rodrigues \\ Pauliana Leão de Souza' \\ Érica Antunes Jimenez' \\ 1 Universidade do Estado do Amapá, Laboratório \\ de Biologia Pesqueira e Beneficiamento do \\ Pescado \\ Correspondência / Correspondence \\ Marilu Teixeira Amaral \\ E-mail:mariamaral0824@gmail.com
}

\section{Resumo}

O mercado consumidor cada vez mais exige produtos inovadores e de qualidade, e para suprir tal demanda é necessário que experimentos com diferentes produtos sejam realizados. A análise sensorial é um método rápido e eficaz para avaliar a aceitabilidade de novos produtos no mercado de alimentos. Diante disso, o presente trabalho procurou avaliar o grau de aceitação do fishburger de Acará-açu no mercado macapaense. A análise sensorial foi feita por 145 degustadores não treinados e selecionados aleatoriamente, os quais atribuíram notas de 1 a 9 ( 1 =desgostei extremamente; 9 =gostei extremamente), segundo escala hedônica, para os seguintes atributos: sabor, aroma, textura, cor, teor de sal e aceitação geral. Para a intenção de compra as notas atribuídas pelos degustadores variaram de 1 a $5(1=$ certamente não compraria o produto; $5=$ certamente compraria o produto). A partir das características sensoriais observadas neste estudo, percebeu-se que as amostras de fishburger de Acará-açu submetidos à análise sensorial obtiveram índice de aceitabilidade superior a $82 \%$ para todos os atributos estudados e a intenção de compra foi confirmada por $80 \%$ dos provadores, o que configura indicativo da viabilidade da produção desse produto e distribuição no mercado de alimentos.

Palavras-chave: Análise sensorial. Tecnologia de Alimentos. Intenção de compra. 


\section{Abstract}

The increasing consumer market requires innovative and quality products to respond to demand; thereby it is necessary that new experiments of new products are performed. Furthermore, a sensory analysis is a quick and an effective method to evaluate the acceptability of new food products. Therefore, the present study sought to assess the degree of acceptance of the fish burger meat of Acará-açú in the Macapá market. A sensory analysis was performed by 145 taste-testers, randomly selected and without prior training, of whom scored 6 parameters on a hedonic scale between 1 and 9, assigning score 1 for "extremely disliked" and 9 for "greatly enjoyed". The following parameters were tested: taste, aroma, texture, color, salt content and general acceptance. In addition, the taste-testers were asked to score the likelihood that they would purchase the food product if available. The scores attributed by tasters ranged from 1 to 5 , considering 1 for "I definitely would not buy the product" and 5 for "I would certainly buy the product". From the sensory characteristics observed in this study, we may conclude that the fish burger's sensory analysis showed acceptability index greater than $82 \%$ for all attributes and the purchase intention was confirmed by $80 \%$ of the attendees. The tallied scores averaged above 5 for each sensory parameter indicating that fish burger meat of Acará -açú taste-tested in the Macapa market received positive acceptance which Indicative of the viability of the production of this product and distribution in the food market.

Key words: Sensory analysis. Food Technology. Purchase Intent.

\section{Introdução}

O pescado é um importante componente da alimentação humana, entretanto, pode perder rapidamente suas características sensoriais por ser uma matéria-prima altamente perecível. ${ }^{1} \mathrm{O}$ consumo de peixes e crustáceos é um dos segmentos alimentícios de mais rápido crescimento no Brasil, obteve uma taxa de crescimento de $9 \%$ nos últimos seis anos, ultrapassando outras proteínas de origem animal. ${ }^{2}$

Para superar a curta vida útil e aproveitar ao máximo a matéria-prima, técnicas de processamento do pescado podem ser utilizadas; dentre elas, a formulação de fishburger é considerada uma técnica inovadora e pouco conhecida no mercado. 
A elaboração de produtos pesqueiros contribui para agregar valor ao produto final, pois, para a produção de fishburger, podem ser utilizados resíduos do processamento e espécies de baixo valor comercial. Através do uso dessas técnicas, podemos obter produtos de qualidade adequados à demanda do consumidor moderno. ${ }^{3}$

Para avaliar a qualidade sensorial dos alimentos, é necessário estudar a aceitação dos consumidores e então identificar como as características sensoriais do produto elaborado influenciam na escolha. ${ }^{4} \mathrm{~A}$ análise sensorial é realizada em função das respostas transmitidas pelos indivíduos às várias sensações que se originam de reações fisiológicas em resposta a estímulos, gerando a interpretação das propriedades intrínsecas dos produtos. Para isso, é preciso que haja entre as partes, indivíduos e produtos, contato e interação. ${ }^{5}$

A análise de aceitação possibilita a obtenção de informações importantes, refletindo o grau com que os consumidores gostam ou desgostam de um determinado produto. ${ }^{6}$ Diante do exposto, este trabalho objetiva elaborar fishburger à base de Acará-açu, realizar análise sensorial e avaliar a aceitabilidade junto ao mercado consumidor do município de Macapá-AP.

\section{Metodologia}

Os exemplares de Acará-açu utilizados nesse experimento foram adquiridos eviscerados frescos em supermercados locais. Para verificar o teor de frescura da carne do pescado a ser processado foi aplicado o formulário de acordo com a metodologia proposta por Hootman. ${ }^{7}$

As amostras adquiridas para processamento apresentaram caracteres organolépticos em conformidade com o que é recomendado pelo Regulamento Técnico de Identidade e Qualidade de Peixe Fresco (Inteiro e Eviscerado), ${ }^{8}$ considerando-se a aparência geral, pele, escamas, olhos, brânquias, mucosidade, opérculo, elasticidade dos músculos, odor, cor e sabor característicos da espécie.

Assim que adquiridos, os exemplares de Acará-açu foram transportados para o laboratório de Biologia Pesqueira e Tecnologia do Pescado da Universidade do Estado do Amapá (UEAP). A espécie Acará-açu (Lobotessurinamensis) foi escolhida porque, apesar de sua carne apresentar um elevado potencial culinário, a população macapaense ainda não possui o hábito de consumir peixes marinhos e poucos estudos foram desenvolvidos para avaliar o seu potencial na fabricação de produtos processados. 
Para a elaboração do fishburger, foram utilizados os seguintes ingredientes: polpa de pescado, água, sal, fécula de mandioca e condimento próprio para hambúrguer. Depois de obtida a polpa, foram adicionados os ingredientes supracitados. As quantidades em gramas utilizadas no processo foram sumarizadas na Tabela 1. Após a mistura dos ingredientes, os fishburgers foram moldados, embalados e congelados em freezer a uma temperatura de $-18^{\circ} \mathrm{C}$ (Figura 1 ).

Tabela 1. Ingredientes utilizados na formulação de fishburger de Acará-açu no mercado macapaense, Amapá, 2013.

\begin{tabular}{lc}
\hline \multicolumn{1}{c}{ Ingredientes } & Quantidades \\
\hline Polpa de acará-açu & $1 \mathrm{~kg}$ \\
\hline Água & $15 \mathrm{ml}$ \\
\hline Sal comum & $5 \mathrm{~g}$ \\
\hline Fécula de mandioca & $100 \mathrm{~g}$ \\
\hline Tempero misto & $10 \mathrm{~g}$ \\
\hline
\end{tabular}




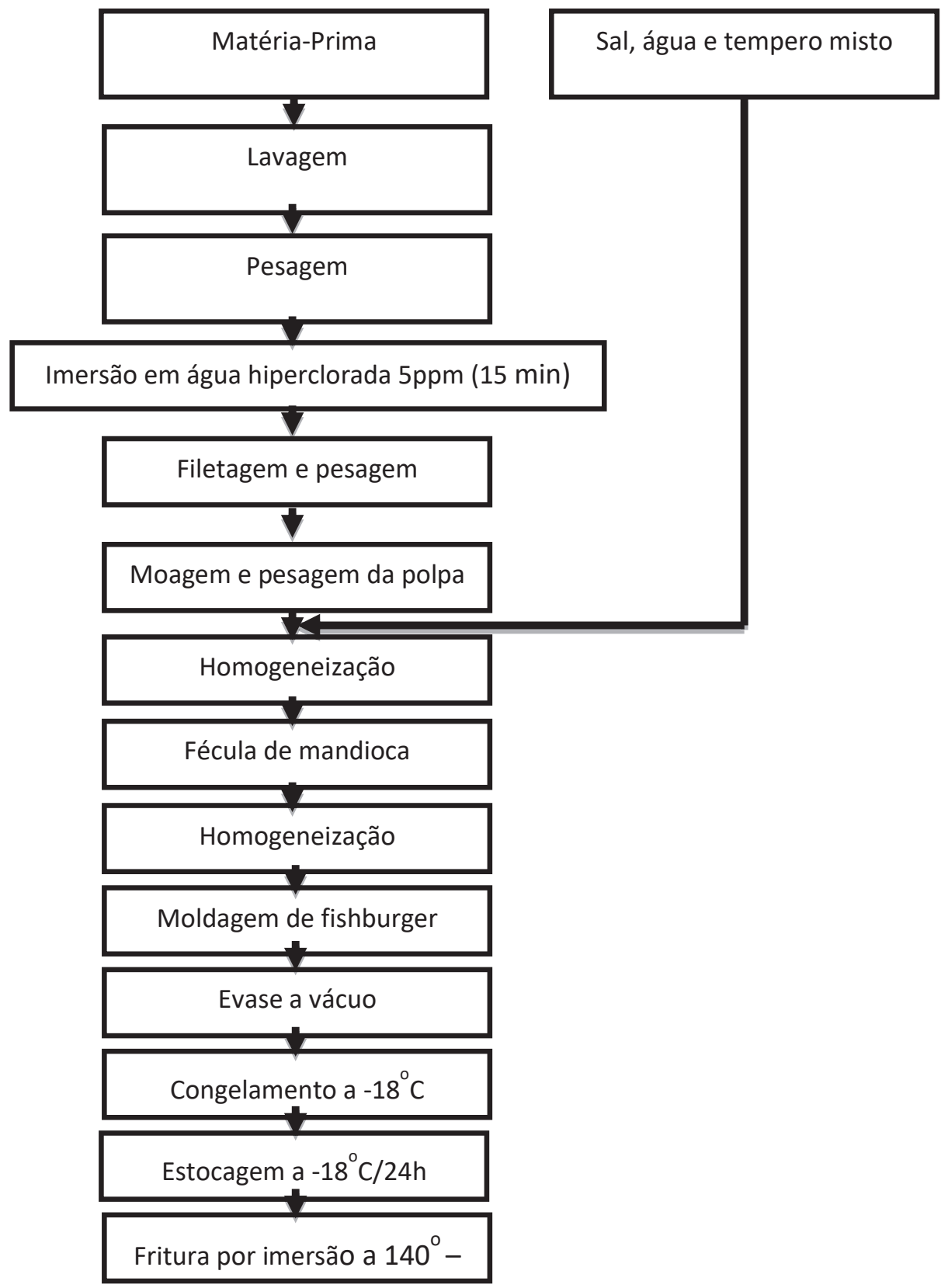

Figura 1. Fluxograma do processamento de fishburger de Acará-Açu, Macapá, Amapá, 2013. 
Posteriormente, os fishburgers passaram pelo processo de fritura. Nesse processo, os hambúrgueres foram imersos em óleo vegetal em uma temperatura que variou entre $140^{\circ}$ a $170^{\circ} \mathrm{C}$ durante 5 minutos. Após esse procedimento, os produtos elaborados foram encaminhados para análise sensorial.

A análise sensorial foi realizada durante a 50aㅡ Expofeira Agropecuária do Amapá, no período de 27 de setembro a 6 de outubro de 2013, no Parque de Exposições da Fazendinha, distrito de Macapá-AP. As análises foram feitas no estande destinado à Tecnologia do Pescado durante todo o evento entre $18 \mathrm{~h}$ e $20 \mathrm{~h}$.

O produto fishburger de Acara-açu foi avaliado sensorialmente por 145 degustadores não treinados e selecionados aleatoriamente. Os degustadores escolhidos apresentaram idade variando entre 18 e 56 anos, $63 \%$ são do sexo masculino, 55\% dos entrevistados são naturais do Estado do Amapá e apresentam renda mensal em torno de três salários mínimos.

Para análise sensorial, foi utilizada a escala hedônica estruturada de 9 pontos, onde: $1=$ desgostei extremamente; $9=$ gostei extremamente, para os atributos: sabor, aroma, textura, cor, teor de sal e aceitação geral. Para a intenção de compra, foi aplicada a escala estruturada de 5 pontos, onde: $1=$ certamente não compraria o produto; $5=$ certamente compraria o produto.

A ficha do teste sensorial foi elaborada de acordo com a metodologia proposta por Dutcosky, ${ }^{9}$ utilizada por Veit et al. ${ }^{10}$ Os dados obtidos foram tabelados em planilha eletrônica para posterior análise estatística descritiva. O índice de aceitabilidade foi calculado através da expressão matemática IA\% = X.100/N, onde X representa a média de cada amostra e N a nota máxima de cada amostra dada pelos provadores. O critério de corte utilizado para o índice ser considerado de boa aceitação foi igual ou superior a $70 \% .^{10}$

\section{Resultados e discussão}

A partir das características sensoriais observadas neste estudo, observou-se que as amostras de fishburger de Acará-açu submetidas à análise sensorial obtiveram escores de aceitação entre 5 e 9 para os atributos: cor, aroma, sabor, textura, teor de sal e aceitação geral. A frequência referente à nota "gostar" foi superior a 96,55\% para todos os atributos estudados e inferior a $5 \%$ para o grau de indiferença, indicando uma boa aceitação do produto (Figura 2). 


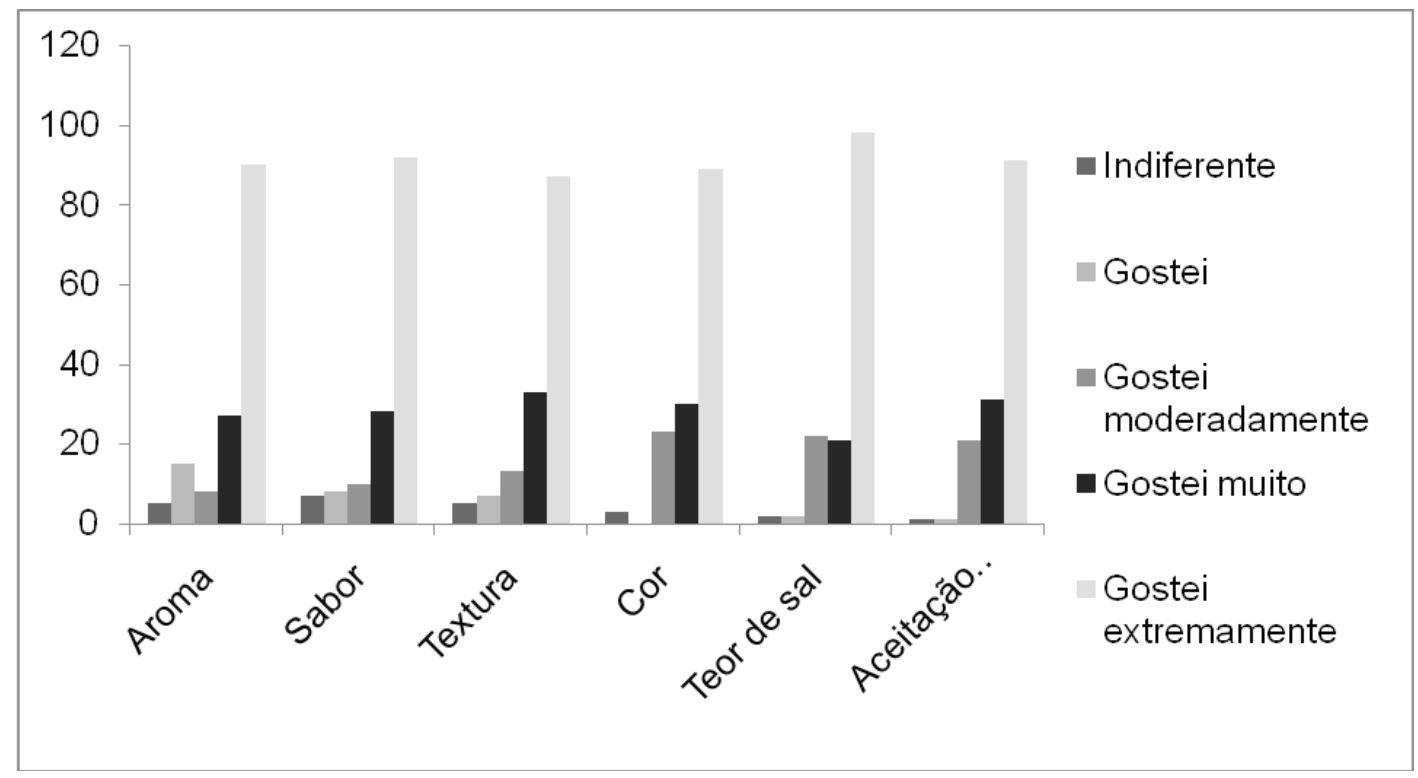

Figura 2. Avaliação dos consumidores macapaenses aos atributos sensoriais do fishburger de Acará-açu, Amapá, 2013.

A análise sensorial é um método importante na avaliação da qualidade e aceitação de um novo produto alimentício, pois nenhuma análise química pode substituir os receptores sensoriais que irão apontar as preferências dos consumidores. De acordo com Vieira et al., ${ }^{10}$ os métodos objetivos podem medir fatores indicativos de oxidação, composição química e grau de decomposição, mas apenas a avaliação sensorial quantifica a percepção e intensidade de aceitação do produto pelos consumidores.

Vieira et al., ${ }^{10}$ relatam que os principais fatores de processamento influenciam as propriedades sensoriais da carne do pescado, principalmente sabor, aroma e textura. Lima e colaboradores, ${ }^{11}$ ao elaborar fishburger de tilápia do Nilo, encontraram resultados semelhantes, identificando aceitação de mercado do produto oferecido, apresentando-se entre moderadamente e muito aceito.

O teste de aceitabilidade IA\% revelou o potencial de aceitação do produto testado, no qual todos os atributos analisados apresentaram média superior a 7 e IA\% superior a $80 \%$, ficando acima da norte de corte estipulada em $70 \%$ (Tabela 2). 
Tabela 2. Índice de aceitabilidade IA\% e média dos atributos sensoriais de fishburger de Acará-açu. Macapá, Amapá, 2013.

\begin{tabular}{lcc}
\hline \multicolumn{1}{c}{ Atributos } & IA\% & Média \\
\hline Aroma & 95,6 & 8,6 \\
Sabor & 94,4 & 8,5 \\
Textura & 96,7 & 8,7 \\
Cor & 91,1 & 8,2 \\
Teor de sal & 82,2 & 7,4 \\
Aceitação geral & 90,0 & 8,1 \\
\hline
\end{tabular}

Não foram encontrados estudos de aceitação e análise sensorial na literatura com a espécie $L$. surinamensis, no entanto, resultados similares ao do presente trabalho foram relatados por Sales et al., ${ }^{12}$ que testaram a aceitação sensorial em escala hedônica de 9 pontos em caranhas e obtiveram a média dos escores de aceitação igual a 8, situada no termo hedônico "gostei muito", indicando que tal produto apresenta boa aceitação. Segundo Nielsen et al., ${ }^{13} 75 \%$ de consumidores de salmão norueguês consideram a textura como atributo de qualidade de maior destaque.

As médias das notas para o parâmetro intenção de compra, que variaram de 3 a 5 , apontam resultados entre: talvez comprasse/talvez não comprasse e decididamente compraria o produto. O teste de intenção de compra mostrou que mais de $80 \%$ dos consumidores que participaram do teste tinham a intenção de comprar o produto caso o encontrasse à venda (Figura 3). 


\section{Número de compradores}

\section{Certamente não compraria}

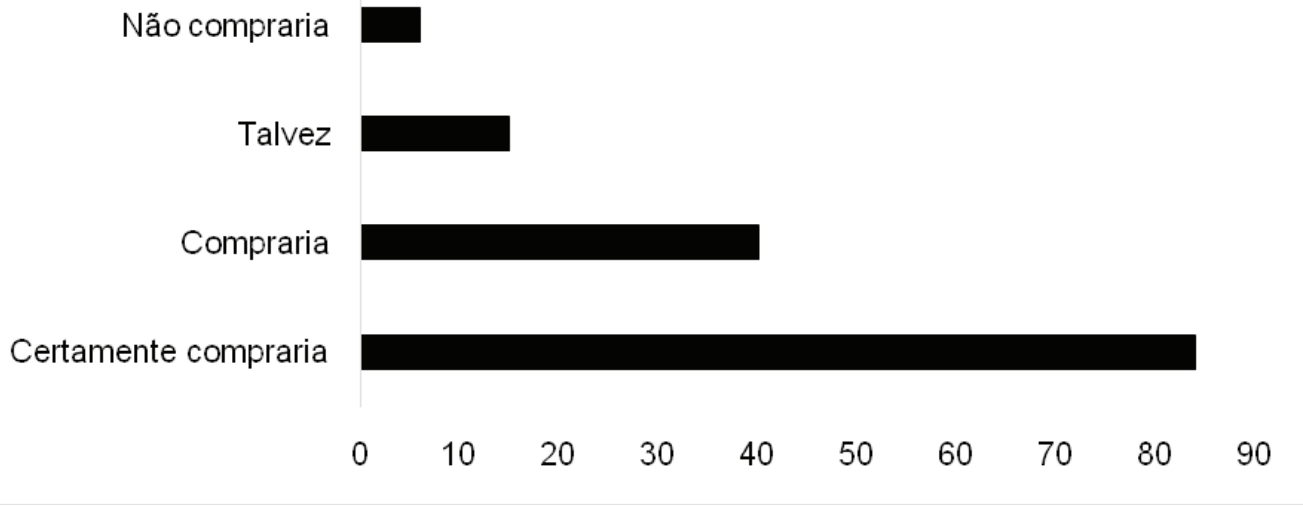

Figura 3. Avaliação de intenção de compra do produto tipo fishburger de Acará-açu, Macapá, Amapá, 2013.

Dutra e Costa ${ }^{14}$ apresentaram duas formulações de hambúrgueres à base de tilápia e obtiverem uma boa aceitação (> 75\%) de acordo com a avaliação de intenção de compra feita. Silva e colaboradores ${ }^{15}$ avaliaram a intenção de compra de 40 consumidores em relação ao fishburger de tilápia e tiveram como resultado: 32,5\% dos provadores informaram que certamente comprariam o produto, $45 \%$ comprariam o produto, $20 \%$ talvez comprariam e 2,5 \% não comprariam e $0 \%$ certamente não comprariam.

Os produtos elaborados à base de pescado na região Norte ainda são pouco conhecidos, os experimentos com fishburger comumente encontrados na literatura são direcionados para a tilápia, pois esta possui um importante papel no mercado brasileiro de alimentos, visto que, segundo dados estatístico do MPA, ${ }^{2}$ a espécie é a mais cultivada no Brasil.

Pesquisas que mostram a viabilidade da formulação de produtos e subprodutos à base de pescado são importantes, ${ }^{3,4,6,9,16,17}$ uma vez que difundem a ideia de inserção desse alimento rico em proteínas, minerais e ácidos nucleicos na dieta dos brasileiros através da elaboração de produtos inovadores feitos com carne de peixes. Além disso, são indicativos econômicos, pois apontam para o potencial de mercado dos novos produtos, atestando que estes apresentam condições de competir igualmente com outros de origem animal que já estão no mercado há mais tempo. 


\section{Conclusão}

A análise sensorial é importante fator na avaliação da qualidade e estabilidade de um produto alimentício, pois nenhum teste instrumental ou químico pode substituir os receptores sensoriais. Embora métodos objetivos meçam fatores indicativos de oxidação, como produtos de decomposição, apenas a avaliação sensorial quantifica a percepção total de intensidade de sabor ou qualidade do produto.

Os principais fatores de processamento que influenciam as propriedades sensoriais de carne de pescado, principalmente sabor, aroma e textura, são qualidade da matéria-prima, fração de peixe utilizada para elaboração do produto e condições de estocagem do produto congelado.

Dessa maneira, os resultados obtidos no presente estudo sugerem uma possibilidade de aproveitamento da carne do Acará-açu para elaboração de fishburgers, visto que as características sensoriais avaliadas foram bem aceitas pelos entrevistados.

\section{Referências}

1. Santos CAML. A qualidade do pescado e a segurança dos alimentos. Anais do II Simpósio de Controle de Qualidade do Pescado; 6-8 jun. 2006, São Vicente, São Paulo: Boletim do Instituto de Pesca; 2006. p. 1-6. Disponível em: ftp://ftp.sp.gov.br/ftppesca/qualidade_pescado.pdf

2. Brasil. Ministério da Pesca e Aquicultura. Boletim estatístico da pesca e aquicultura. Brasília: MPA; 2012. 60 p. Disponível em: http://www.mpa.gov.br.

3. Amaral M. T. Elaboração de produtos processados a partir da carne de mapará (Hypophthalmus edentatus). Anais do V Simpósio de Controle de Qualidade do Pescado; 19-21 jun. 2012; Santos, São Paulo: Boletim do Instituto de Pesca; 2012. p 1-6.

4. Hough G, Wakeling I, Mucci A, Chambers IV E, Gallardo IM, Alves LR. Number of consumers necessary for sensory acceptability tests. Food Quality and Preference 2006; 17(6):522-526.

5. Instituto Adolfo Lutz. Métodos físico-químicos para análise de alimentos. São Paulo: IAL; 2008. p. $161-278$.

6. Westad F, Hersleth M, Lea P. Strategies for consumer segmentation with application on preference data. Food Quality and Preference 2004; 15(2):681-687.

7. Hootman RC. Manual on descriptive analysis testing for sensory evaluation. Philadélphia: Americcan Society for Testing and Materials; 1992. (ASTM manual Series: MNL 13).

8. Brasil. Portaria no 185, de 13 de maio de 1997. Regulamento Técnico de Identidade e Qualidade de Peixe Fresco (Inteiro e Eviscerado). Diário Oficial da União 19 maio 1997.

9. Dutcpsky SD. Análise sensorial de alimentos. 2ºd. Curitiba: Champagnat; 1996. 239 p. 
10. Veit JC, Signor AA, Reis ES, Freiden A, Moore OQ, Boscolo WR. Análise sensorial de filés fritos ou defumados de jundiás submetidos a alimentação com certificação orgânica ou comercial. Revista Varia Scientia Agrárias Engenharia de Pesca 2013; 3(1):151-160.

11. Vieira LA, Aranha BC, Viana CE, Epping NC, Prestes OD, Augusti PR. Elaboração de Fishburguer à base de palometa (Serrasalmuss pilopleura) e avaliação da aceitabilidade e intenção de compra. Anais do Salão Internacional de Ensino, Pesquisa e Extensão 2012; 4(2):2-4.

12. Lima JS, Araujo JM, Dias SS, Silva EA, Silva EAL, Aquino AB, et al. Análise microbiológica e sensorial de Fishburger elaborado com tilápia do Nilo (Oreochromis niloticus) com adição de conservantes naturais. GEINTEC - Gestão, Inovação e Tecnologia 2014; 4(1):560-567.

13. Sales PVG. Avaliação sensorial de duas formulações de hambúrguer de peixe. Revista Brasileira de Produtos Agroindustriais 2015; 17(1):17-23.

14. Nielsen D, Hyldig G, Nielsen J, Nielsen HH. Sensory properties of marinated herring (Clupea harengus) processed from raw material comercial landings. Journal of the Science of Food and Agriculture 2005: 85(1):127-134.

15. Dutra IR, Costa DPS. Processamento e avaliação sensorial de hambúrguer de tilápia. Revista Higiene Alimentar 2011; 25(94/95):1-3.

16. Silva JL. Elaboração e intenção de compra de hambúrguer de peixe com aplicação de espessante natural. Anais do VII Congresso Norte Nordeste de Pesquisa e Inovação; 19-21 out. 2012; Palmas, Tocantins: IFTO; 2012. p 1-8

17. Silveira LS, Abdallah PR, Hellebrandt L, Barbosa MN, Feijó FT. Análise socioeconômica do perfil dos consumidores de pescado no município de Rio Grande. SINERGIA 2012; 16(1):9-19. 
